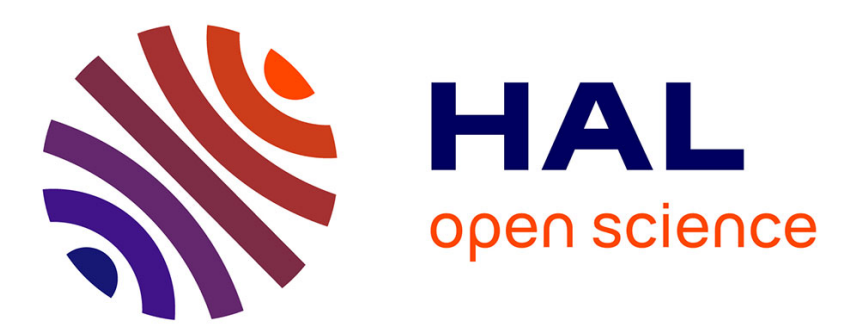

\title{
Damping properties of bi-dimensional sandwich structures with multi-layered frequency-dependent visco-elastic cores
}

Jean Ayodele Adessina, Mohamed Hamdaoui, Chao Xu, El Mostafa Daya

\section{To cite this version:}

Jean Ayodele Adessina, Mohamed Hamdaoui, Chao Xu, El Mostafa Daya. Damping properties of bi-dimensional sandwich structures with multi-layered frequency-dependent visco-elastic cores. Composite Structures, 2016, 154, pp.334-343. 10.1016/j.compstruct.2016.07.056 . hal-03232790

\author{
HAL Id: hal-03232790 \\ https://hal.science/hal-03232790
}

Submitted on 11 Jun 2021

HAL is a multi-disciplinary open access archive for the deposit and dissemination of scientific research documents, whether they are published or not. The documents may come from teaching and research institutions in France or abroad, or from public or private research centers.
L'archive ouverte pluridisciplinaire HAL, est destinée au dépôt et à la diffusion de documents scientifiques de niveau recherche, publiés ou non, émanant des établissements d'enseignement et de recherche français ou étrangers, des laboratoires publics ou privés. 


\title{
Damping properties of bi-dimensional sandwich structures with multi-layered frequency-dependent visco-elastic cores
}

\author{
Ayodele Adessina $^{\mathrm{a}}$, Mohamed Hamdaoui ${ }^{\mathrm{b}}, \mathrm{Chao}_{\mathrm{Xu}}^{\mathrm{d}}$, El Mostafa Daya ${ }^{\mathrm{b}, \mathrm{c}}$ \\ ${ }^{a}$ Ecole Nationale des Ponts et Chaussées, Laboratoire Navier UMR 8205, \\ 6-8 avenue Blaise Pascal, \\ F-77455 Marne-la-Vallée cedex 2 \\ $\mathrm{Tel}: 0164153737$ \\ Fax : 0164153741 \\ ${ }^{b}$ Université de Lorraine, LEM3 UMR 7239, \\ Ile du Saulcy \\ F-57045 Metz - Cedex 01 \\ $\mathrm{Tel}: 03-87-31-54-77$ \\ Fax : 03-87-31-53-66 \\ ${ }^{c}$ Labex DAMAS, Université de Lorraine, \\ Ile du Saulcy \\ F-57045 Metz - Cedex 01 \\ $\mathrm{Tel}: 03-87-31-53-70$ \\ Fax : 03-87-31-53-66 \\ ${ }^{d}$ School of Astronautics, Northwestern Polytechnical University, \\ $X i$ 'an 710072, China, \\ $\mathrm{Tel}:$ 86-29-88493620 \\ Fax : 86-29-88493620
}

\begin{abstract}
The aim of this paper is to present a finite element model based on first order shear theory (zig-zag approach) to compute the damping characteristics of sandwich structures with multi-layered frequency-dependent viscoelastic cores. The model is validated versus a layerwise finite element model and used to study damping and rigidity of a laminated glass configuration with a multi-layered visco-elastic core composed of acoustic PVB and PVB. It is shown that the rigidity index of the structure (defined as the adimensionalized inverse of the maximal transverse displacement under a static load) evolves linearly with the viscoelastic layers' thicknesses and quadratically versus elastic layer's thickness. The first mode damping and resonant frequency show a non monotonous behaviour. In particular, the existence of an optimal faces thickness for damping is shown while a quadratic behaviour of frequency versus acoustic PVB layer thickness is reported.

Keywords: Sandwich beam, Damping, Rigidity, Multilayered viscoelatic core, Laminated glass
\end{abstract}

Email address: mohamed.hamdaoui@univ-lorraine.fr (Mohamed Hamdaoui) 


\section{Introduction}

Viscoelastic sandwich structures are commonly used as damping devices in many domains such as aeronautics, aerospace, mechanical and civil engineering. They play a key role in vibration control, shocks absorption and noise reduction. They are made of two identical elastic and stiff layers separated by at least one soft viscoelastic layer. Due to the difference between in-plane displacements of the elastic layers and to the low viscoelastic core stiffness, the latter experiences a strong shear which is known to be responsible of the damping properties of viscoelastic sandwich structures. The study of sandwich structures started in 1959 with the work of Kerwin [1] that has been further improved by Ungar [2] in 1962. Analytical methods were first developed to estimate damping properties (loss factor and resonant frequencies) of sandwich beams and plates with simple boundary conditions. These can be found in the works of DiTaranto and Blasingame [3], Mead and Markus [4] and Rao [5]. Since then, many investigations have been devoted to vibration analysis of viscoelastic structures and especially damping properties computation. In the finite elements framework, several works in the literature have been devoted to model viscoelastic structures[6],[7],[8],[9],[10],[11],[12]. However, modelling frequency dependent viscoelastic sandwich structures implies dealing with nonlinear problems because of the frequency dependence of the stiffness matrix. Unfortunately, legacy finite elements codes such as Abaqus or Ansys are not able to solve these non-linear problems efficiently. Numerical methods such as the modal strain energy method [13] and the asymptotic numerical method [14] have been developed in order to solve the resulting nonlinear problem. An overview of the different methods of resolution can be found in [15]. Recently, the research has focused on multilayer or laminated sandwich structures for their improved multifunctional properties in terms of mechanical damping, rigidity, acoustics, etc. Araujo et al. [16] presented a finite element model for damping analysis of anisotropic laminated viscoelastic sandwich structures. Li and Narita [17] studied the optimal design of symmetrical laminated thin plates comprised of fiber-reinforced layers and viscoelastic layers by varying the material fibers orientation. Nguyen et al. [18] developed a higher-order zig-zag theory for viscoelastic laminated composite plates for efficient temporal response computation. Yang et al. [19] performed vibration analysis of multilayer thick sandwich cylindrical shells with a viscoelastic core under arbitrary boundary conditions investigating the effect of fiber orientation angle and thickness. Akoussan et al. [20] presented a finite element model for multilayer orthotropic viscoelastic sandwich structures along with a resolution method based on asymptotic numerical method. Bouayed and Hamdi [21] proposed a shell finite element model for vibroacoustic studies of multilayers car windscreens while providing a good review of the past finite element models for multilayers structures. However, to the author's best knowledge, most of the studies that can be found in the literature on multilayer viscoelastic 
sandwich structures do not consider the possibility of having multi-layered viscoelastic cores made of different types of viscoelastic materials. For example, windscreens vibro-acoustical damping properties can be enhanced by using multi-layer viscoelastic layers made of a plastic layer sandwiched by two PVB layers [22]. Furthermore, the noise damping properties of security laminated glass can be enhanced by sandwiching a high stiffness polymer layer by two low stiffness but high damping polymer layers [23]. In the present work, the authors propose a finite element model based on the zig-zag approach to model a five layered sandwich beam with a viscoelastic core made of two different layers of viscoelastic materials. The model is thought as a tool to study different properties of sandwich structures with multi-layered frequency dependent viscoelastic cores. An application to a five layered laminated glass is considered and the impact of the relevant parameters studied. In section 2, the model kinematics along with the assumptions made are presented. Then, in section 3 , the variational formulation of the problem using the principle of virtual work is derived. In section 4 the finite element model is detailed along with the resolution technique. In section 5 , the finite element model is validated for frequency dependent and frequency independent viscoelastic cores. In section 6 , the model is used to compare some relevant design characteristics (damping, frequency and rigidity) of a laminated glass structure with a multi-layered viscoelastic core.

\section{Kinematic model}

The five layered sandwich structure is assumed to be symmetric as depicted on Figure 1. The first layer is elastic of thickness $h_{f}$, the second layer is viscoelastic of thickness $h_{c_{1}}$ and the central layer is viscoelastic of thickness $h_{c_{2}}$. By symmetry, the fourth layer is viscoelastic of thickness $h_{c_{1}}$ and the fifth layer is elastic of thickness $h_{f}$. Strain and displacement fields

in each layer described by a "zig-zag" model has been adopted: the displacement field in the elastic layer derives from an Euler-Bernoulli approach whereas a Timoshenko model characterizes strain and displacement field in the viscoelastic layers. The present analysis is based on the following assumptions [24, 25]:

- All the materials are assumed linear, homogeneous and isotropic.

- There is no shear in the elastic layers.

- The transverse displacement is assumed to be the same for all plies.

- The Young's modulus of the viscoelastic layers is complex frequency dependent, but the Poisson ratio is assumed to be constant.

- No slipping occurs at the interfaces between the different layers. 
- The elastic layers have the same Young's modulus and mass density.

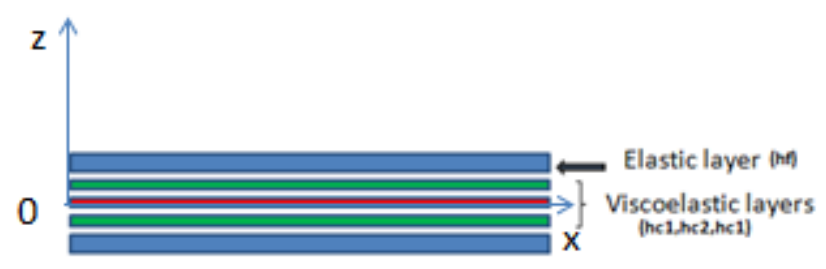

Figure 1: Five layered sandwich beam structure

In the following, $x$ denotes the longitudinal coordinate, $z$ the vertical coordinate and $t$ the time. In all the paper, we shall use the following convention $\frac{\partial g}{\partial x}=g^{\prime}$ and $\frac{\partial g}{\partial t}=\dot{g}$, with $g$ a function of $(x, t)$. Regarding all the above assumptions, the displacement field and associated deformation in the elastic layer can be expressed as:

$$
\begin{aligned}
U_{i}(x, z, t) & =u_{i}(x, t)-\left(z-z_{i}\right) w^{\prime} \\
W_{i}(x, z, t) & =w(x, t), i=1,5 \\
\varepsilon_{i}(x, z, t) & =u_{i}^{\prime}-\left(z-z_{i}\right) w^{\prime \prime}
\end{aligned}
$$

where $u_{i}$ is in-plane displacement of the $\mathrm{i}^{\text {th }}$ layer and $w$ the common transverse displacement. On the other hand, the displacement field and associated deformation in the viscoelastic layer can be expressed according to Timoshenko theory :

$$
\begin{aligned}
U_{i}(x, z, t) & =u_{i}(x, t)+\left(z-z_{i}\right) \beta_{i}(x, t), \\
W_{i}(x, z, t) & =w(x, t), i=2,3,4 \\
\varepsilon_{i}(x, z, t) & =u_{i}(x, t)^{\prime}+z \beta_{i}(x, t)^{\prime} \\
\xi_{i}(x, z, t) & =\beta_{i}(x, t)+w(x, t)^{\prime}
\end{aligned}
$$

where $\xi_{i}$ is the shear strain, $\beta_{i}$ is the rotation of the mid-plane of a viscoelastic layer $i$. The second viscoelastic layer in the structure's core is taken as the central layer $u_{3}=u$. Furthermore, requiring the continuity of the displacement field at the interfaces between the 
different layers results in the following relationship:

$$
\begin{aligned}
& u_{1}=u_{2}+\frac{h_{c_{1}} \beta_{2}-h_{f} w^{\prime}}{2} \\
& u_{2}=u_{3}+\frac{h_{c_{2}} \beta_{3}+h_{c_{1}} \beta_{2}}{2} \\
& u_{4}=u_{3}-\frac{h_{c_{2}} \beta_{3}+h_{c_{1}} \beta_{4}}{2} \\
& u_{5}=u_{4}-\frac{h_{c_{1}} \beta_{4}+h_{f} w^{\prime}}{2} .
\end{aligned}
$$

By setting $u_{3}=u$ and using the symmetry hypothesis (which gives $\beta_{2}=\beta_{4}$ ), the above displacement field can be rewritten as a function of the displacement of the central layer:

$$
\begin{aligned}
& u_{1}=u+h_{c_{1}} \beta_{2}+\frac{h_{c_{2}} \beta_{3}-h_{f} w^{\prime}}{2}, \\
& u_{2}=u+\frac{h_{c_{1}} \beta_{2}+h_{c_{2}} \beta_{3}}{2}, \\
& u_{4}=u-\frac{h_{c_{2}} \beta_{3}+h_{c_{1}} \beta_{2}}{2}, \\
& u_{5}=u-h_{c_{1}} \beta_{2}-\frac{h_{c_{2}} \beta_{3}-h_{f} w^{\prime}}{2} .
\end{aligned}
$$

\section{Variational formulation}

The virtual work principle is applied to establish the equation of motion of the five layered sandwich beam. The virtual work principle components of a symmetric five layered sandwich beam can be expressed as the sum of the different virtual works of all the layers:

- In the elastic layers

$$
\int_{0}^{L}\left(N_{i} \delta u_{i}^{\prime}+M_{i} \delta w^{\prime \prime}\right) d x=-\rho_{f} S_{f} \int_{0}^{L} \ddot{w} \delta w d x
$$

- In the visco-elastic layers

$$
\int_{0}^{L}\left(N_{i} \delta u_{i}^{\prime}+M_{i} \delta \beta_{i}^{\prime}+T_{i}\left(\delta \beta_{i}+\delta w^{\prime}\right)\right) d x=-\rho_{c_{i}} S_{c_{i}} \int_{0}^{L} \ddot{w} \delta w d x
$$

where $N_{i}, M_{i}$ are the axial forces and the bending moment of each layer, $T_{i}$ is the shear force in a layer $i$, whose expressions are given in Appendix .2. The quantities $\rho_{c_{i}}$ and $S_{c_{i}}$ represent the density and cross sectional area of viscoelastic layers $i=(2,3,4)$, respectively. We denote $\left(\delta u, \delta \beta_{i}, \delta w\right)$ the generalized displacement vector. The total virtual work of the 
sandwich beam can be expressed as :

$$
\begin{aligned}
\int_{0}^{L}\left(N \delta u^{\prime}+M_{\beta_{2}} \delta \beta_{2}^{\prime}+M_{\beta_{4}} \delta \beta_{4}^{\prime}+M_{\beta_{3}} \delta \beta_{3}^{\prime}\right. & \left.+T_{2} \delta \beta_{2}+T_{4} \delta \beta_{4}+T_{3} \delta \beta_{3}+M_{w} \delta w^{\prime \prime}+T \delta w^{\prime}\right) d x \\
& =-\left(2 \rho_{f} S_{f}+2 \rho_{c_{2}} S_{c_{2}}+\rho_{c_{3}} S_{c_{3}}\right) \int_{0}^{L}(\ddot{w} \delta w d x)
\end{aligned}
$$

with:

$$
\begin{aligned}
N & =\sum_{i=1}^{5} N_{i}, \\
M_{\beta_{2}} & =M_{2}+\frac{h_{c_{1}}}{2}\left(2 N_{1}+N_{2}\right), \\
M_{\beta_{3}} & =M_{3}+\frac{h_{c_{2}}}{2}\left(N_{1}+N_{2}-N_{4}-N_{5}\right), \\
M_{\beta_{4}} & =M_{4}-\frac{h_{c_{1}}}{2}\left(N_{4}+2 N_{5}\right), \\
M_{w} & =M_{1}+M_{5}+\frac{h_{f}}{2}\left(N_{5}-N_{1}\right), \\
T & =T_{2}+T_{3}+T_{4},
\end{aligned}
$$

Equation (14) expresses a coupling between the flexural and the membrane effect. It can be split into two different equations when considering the balance condition:

$$
\int_{0}^{L} N \delta u^{\prime} d x=0
$$

The symmetry hypothesis gives us $\beta_{2}=\beta_{4}, N_{1}=N_{5}, N_{2}=N_{4}, T_{2}=T_{4}, M_{1}=M_{5}$ and $M_{2}=M_{4}$ which yields to $M_{\beta_{2}}+M_{\beta_{4}}=2 M_{2}, M_{\beta_{3}}=M_{3}$ and $M_{w}=2 M_{1}$. The flexural motion of the five layered symmetric sandwich beam is expressed by the following expression:

$$
\begin{array}{r}
\int_{0}^{L}\left(2 M_{2} \delta \beta_{2}^{\prime}+M_{3} \delta \beta_{3}^{\prime}+2 T_{2} \delta \beta_{2}+T_{3} \delta \beta_{3}+2 M_{1} \delta w^{\prime \prime}+T \delta w^{\prime}\right) d x \\
=-\left(2 \rho_{f} S_{f}+2 \rho_{c_{2}} S_{c_{2}}+\rho_{c_{3}} S_{c_{3}}\right) \int_{0}^{L} \ddot{w} \delta w d x .
\end{array}
$$

We remind that equation (16) is a non-linear integro-partial differential equation and since there is no analytical solution for this equation, numerical discretization based on the finite elements method appears as an appropriate way to solve the problem . 


\section{Finite element formulation}

In the case of free vibrations, the solution of equation (16) can be written in the harmonic form:

$$
\begin{aligned}
& w(x, t)=W(x) e^{i \omega t}, \\
& \beta_{2}(x, t)=B_{2}(x) e^{i \omega t}, \\
& \beta_{3}(x, t)=B_{3}(x) e^{i \omega t},
\end{aligned}
$$

One dimensional two nodes finite elements are used in this work. Each nodes has four degrees of freedom describing the transversal displacement $w$, the slope $w^{\prime}$ and the rotations $\beta_{2}, \beta_{3}$ related respectively to the second and third layer of the structure.

Thus, for each element bounded by the nodes 1 and 2, the modal displacement vector is written as follows:

$$
U_{e}=\left[\begin{array}{llllllll}
W^{1} & \left(W^{1}\right)^{\prime} & B_{2}^{1} & B_{3}^{1} & W^{2} & \left(W^{2}\right)^{\prime} & B_{2}^{2} & B_{3}^{2}
\end{array}\right]^{t}
$$

Thanks to the classical polynomial shape functions [26] the displacement field of each element can be expressed as:

$$
\begin{aligned}
& W=\left[N_{w}\right]\left\{U_{e}\right\}, \\
& B_{2}=\left[N_{\beta_{2}}\right]\left\{U_{e}\right\}, \\
& B_{3}=\left[N_{\beta_{3}}\right]\left\{U_{e}\right\},
\end{aligned}
$$

where $\left[N_{w}\right],\left[N_{\beta 2}\right],\left[N_{\beta 3}\right]$ denote the shape functions given in the Appendix .1.

Considering equations (17),(18),(21) combined with the flexural motion (16), one obtains the discretized form of a nonlinear eigenvalue problem expressing free vibrations of a five layered sandwich structure:

$$
\left(\left[K_{e}(\omega)\right]-\omega^{2}\left[M_{e}\right]\right)\{U\}=0,
$$

where $\left[K_{e}(\omega)\right]$, and $\left[M_{e}\right]$ denote respectively the rigidity and mass matrices given in the Appendix .1. These matrices are assembled to get the overall complex non-linear eigenvalue problem

$$
\left([K(\omega)]-\omega^{2}[M]\right)\{U\}=\{0\},
$$

\subsection{Rigidity index}

The rigidity of the structure can be estimated by determining the maximum transverse displacement $W_{\max }$ by solving the standard problem $([K(0)] U=F)$ with $F$ a prescribed 
vector force and $[K(0)]$ the static stiffness rigidity matrix. A rigidity index is given by

$$
R=\frac{h}{W_{\max }},
$$

with $h=2 h_{f}+\left(h_{c_{2}}+h_{c_{3}}+h_{c_{4}}\right)$ the total beam thickness.

\subsection{Asymptotic Numerical Method}

In order to solve equation(23) which is a nonlinear equation, Asymptotic Numerical Method (ANM) has been used. This method consists of expanding the unknown eigenmodes and eigenvectors of equation(23) as a power series with respect to the path $\mathrm{p}$ :

$$
\begin{array}{r}
U=U_{0}+p U_{1}+p^{2} U_{2}+\ldots p^{j} U_{j}+\ldots \\
\lambda=\lambda_{0}+p \lambda_{1}+p^{2} \lambda_{2}+\ldots p^{j} \lambda_{j}+\ldots
\end{array}
$$

where $\lambda=\omega^{2}$. By introducing the above expansions in equation(23)and equating like power of $p$, a serie of linear problem can be obtained. The Diamant approach which will be used in the numerical resolution of the problem is a combination of ANM and differentiation techniques which helps to compute higher order derivatives. The reader can refer to $[15,20,14,27]$ for more details on this method. By applying the ANM to the current problem, a set of two equations concerning the residues can be obtained:

$$
\begin{aligned}
& R(U, \lambda)=\left([K(0)]+E(\lambda)\left[K_{v}\right]-\lambda[M]\right) U \\
& R(U, \lambda)=S(U, \lambda)+T(U, \lambda)
\end{aligned}
$$

where $S(U, \lambda)=([K(0)]-\lambda[M]) U$ and $T(U, \lambda)=E(\lambda)\left[K_{v}\right] U$ with $E_{v}=E(\lambda)+E_{v}(0)$ which is a non-linear function expressing the frequency dependency of the Young and shear modulus of the viscoelastic layer. $\left[K_{v}\right]$ is a purely real matrix. The homotopy technique $[20,14]$ is used to transform the original problem by introducing the path parameter $p$ in order to derive the solution from the real eigenvalue problem $(S(U, \lambda)=0$ ), (a problem which solution $\left(U_{0}, \lambda_{0}\right)$ can be easily computed). The homotopy technique helps to write the new residue by introducing a perturbation $p \in[0,1]$ such as:

$$
R(U, \lambda, p) \equiv S(U, \lambda)+p T(U, \lambda)=0
$$

By expanding $S(U, \lambda)$ and $T(U, \lambda)$ as Taylor series, one can deduce the generic linear problem to be implemented with Diamant [15]. After a suitable initialization and application of the 
chain rule, the resulting equation leads to:

$$
\left(\begin{array}{cc}
A_{0} & U_{0} \\
{ }^{t} U_{0} & 0
\end{array}\right)\left(\begin{array}{c}
U_{j} \\
\kappa
\end{array}\right)=\left(\begin{array}{c}
-S_{j \mid U_{j}=0}-p T_{j \mid U_{j}=0}-T_{j-1} \\
0
\end{array}\right)
$$

where

$$
A_{0}=\left[K(0)-\lambda_{0}[M]+p E\left(\lambda_{0}\right)\left[K_{v}\right]\right.
$$

with $\kappa$ the Lagrange multiplier.

$$
\lambda_{j}=-\frac{{ }^{t} U_{0}\left[-S_{j \mid U_{j}=0}-p T_{j \mid U_{j}=0}-T_{j-1}\right]}{{ }^{t} U_{0}\left[S_{1 \mid U_{1}=0, \lambda_{1}=1}+p T_{1 \mid U_{1}=0, \lambda_{1}=1}\right]}
$$

The solution $(U, \lambda)$ is then determined using the continuation procedure. In order to introduce genericity in the computation of Asymptotic Numerical Method and continuaton process, a toolbox named Diamant based on the described method with automatic differentiation is used. The resolution of nonlinear equation with the toolbox Diamant is exposed in [15].

\section{Model validation}

The model is applied to five layered sandwich structures with different cores (homogeneous and heterogeneous).

\subsection{Structures with a single layer core}

\subsubsection{Constant viscoelastic model}

In this case, the viscoelastic properties of the core are modelled by a complex Young modulus which is assumed to be constant:

$$
E(\omega)=E_{0}\left(1+i \eta_{c}\right)
$$

$E_{0}$ is the Young modulus related to the delayed elasticity and $\eta_{c}$ is the core's loss factor. With this assumptions the eigenvalue problem becomes linear and can be easily solved by the classical methods (QR, Lanczos, etc.). The geometrical and material properties of the five layered sandwich beam are summarized in Table (8) whereas the equivalent frequencies and the associated loss factors are presented in Table (2). In order to compare our results with those of [27], we set $h_{c_{1}}=h_{c_{2}}=\frac{h_{c}}{3}$ with $h_{c}$ the thickness of the viscoelastic layer used in [27]. The linear equivalent frequency and the associated loss factor were computed for different values of the core's loss factor as shown in table 2. One can see that our model 


\begin{tabular}{lcr}
\hline Elastic Faces & Young Modulus & $E_{f}=69 \times 10^{9} \mathrm{~Pa}$ \\
& Poisson ratio & $\nu=0.3$ \\
& Mass density & $\rho_{f}=2766 \mathrm{Kg}^{-3}$ \\
& Thickness & $h_{f}=1.524 \mathrm{~mm}$ \\
\hline Viscoelastic core & Young modulus & $E_{0}=1794 \times 10^{3} \mathrm{~Pa}$ \\
& Poisson ratio & $\nu=0.3$ \\
& Mass density & $\rho_{c}=968.1 \mathrm{Kg} . \mathrm{m}^{-3}$ \\
& Thickness & $h_{c}=0.127 \mathrm{~mm}$ \\
\hline Beam & Length & $L=177.8 \mathrm{~mm}$ \\
& Width & $l=12.7 \mathrm{~mm}$ \\
\hline
\end{tabular}

Table 1: Geometrical and material properties of the clamped-free beam

\begin{tabular}{ccccccc}
\hline Five & layered & beam & & Three & layered & beam. \\
\hline$\eta_{c}$ & Frequency $(\mathrm{Hz})$ & $\eta / \eta_{c}$ & Error & Frequency $(\mathrm{Hz})$ & $\eta / \eta_{c}$ & Error \\
\hline 0.1 & 64.1 & 0.281 & $4.33 \times 10^{-4}$ & 64.1 & 0.281 & $3.28 \times 10^{-4}$ \\
& 296.69 & 0.242 & $1.65 \times 10^{-3}$ & 296.7 & 0.242 & $1.21 \times 10^{-3}$ \\
& 744.48 & 0.154 & $2.16 \times 10^{-3}$ & 744.5 & 0.154 & $1.58 \times 10^{-3}$ \\
& 1395.7 & 0.089 & $2.26 \times 10^{-3}$ & 1395.7 & 0.089 & $1.64 \times 10^{-3}$ \\
& 2264.5 & 0.057 & $2.31 \times 10^{-3}$ & 2264.5 & 0.057 & $1.68 \times 10^{-3}$ \\
& 3349.8 & 0.039 & $2.32 \times 10^{-3}$ & 3349.8 & 0.039 & $1.69 \times 10^{-3}$ \\
0.6 & & & & & & \\
& 65.54 & 0.246 & $4.33 \times 10^{-4}$ & 65.5 & 0.246 & $3.28 \times 10^{-4}$ \\
& 299.2 & 0.232 & $1.65 \times 10^{-3}$ & 299.2 & 0.232 & $1.21 \times 10^{-3}$ \\
& 746.3 & 0.153 & $2.16 \times 10^{-3}$ & 746.3 & 0.153 & $1.58 \times 10^{-3}$ \\
& 1396.6 & 0.089 & $2.26 \times 10^{-3}$ & 1396.6 & 0.089 & $1.64 \times 10^{-3}$ \\
& 2265.2 & 0.057 & $2.31 \times 10^{-3}$ & 2265.2 & 0.057 & $1.68 \times 10^{-3}$ \\
& 3350.2 & 0.023 & $2.32 \times 10^{-3}$ & 3350.2 & 0.023 & $2.32 \times 10^{-3}$ \\
\hline
\end{tabular}

Table 2: Linear frequencies and associated loss factors of the clamped-free sandwich beam. 
perfectly matches the results of [27] in terms of eigenfrequency and damping ratio.

\subsubsection{Frequency dependent viscoelastic models}

A comparison to the three layered beam model of [27] was performed in the case of frequency dependent materials. Polyvinyl-Butyral (PVB) and 3MISD112 were used for the viscoelastic core of the structure.

Core in PVB. We consider elastic faces made in glass separated by three viscoelastic layers in PVB. The frequency dependent of the Young's modulus of the viscoelastic layers is expressed by a power-law:

$$
E(\omega)=E_{\infty}+\left(E_{0}-E_{\infty}\right)\left[1+(i \omega \tau)^{1-\alpha}\right]^{-\beta} .
$$

where $E_{0}=0.479 \times 10^{6} \mathrm{~Pa}$ is the delayed elasticity of the shear modulus, $E_{\infty}=2.35 \times$ $10^{8} \mathrm{~Pa}, \tau=0.3979, \alpha=0.46$ and $\beta=0.1946$. The geometrical and material properties of the sandwich beam glass/PVB/glass are resumed in table 3 . The free vibration frequencies and

\begin{tabular}{lcr}
\hline Elastic Faces & Young Modulus & $E_{f}=64.5 \times 10^{9} \mathrm{~Pa}$ \\
& Poisson ratio & $\nu=0.22$ \\
& Mass density & $\rho_{f}=2737 \mathrm{Kg}^{-3}$ \\
& Thickness & $h_{f}=5 \mathrm{~mm}$ \\
\hline Viscoelastic core & Poisson ratio & $\nu=0.4$ \\
& Mass density & $\rho_{c}=999 \mathrm{Kg} \cdot \mathrm{m}^{-3}$ \\
& Thickness & $h_{c_{1}}=h_{c_{2}}=0.253 \mathrm{~mm}$ \\
\hline Beam & Length & $\mathrm{L}=1 \mathrm{~m}$ \\
& Width & $\mathrm{l}=0.1 \mathrm{~m}$ \\
\hline
\end{tabular}

Table 3: Geometrical and material properties of a clamped-clamped sandwich structure.

the associated loss factor have been computed and the results are compared with that of [27] for validation in table(4). The results of the model match perfectly those of [27].

\begin{tabular}{ccccccc}
\hline Five & layered & beam & & Three & layered & beam \\
\hline Modes & Frequency $(\mathrm{Hz})$ & Loss factor & Error & Frequency $(\mathrm{Hz})$ & Loss factor & Error \\
\hline 1 & 53.74 & $9.11 \times 10^{-3}$ & $4.6 \times 10^{-5}$ & 53.74 & $9.11 \times 10^{-3}$ & $1.38 \times 10^{-3}$ \\
2 & 145.26 & $1.37 \times 10^{-2}$ & $2.6 \times 10^{-5}$ & 145.26 & $1.37 \times 10^{-2}$ & $1.57 \times 10^{-3}$ \\
3 & 278.39 & $1.80 \times 10^{-2}$ & $2.08 \times 10^{-5}$ & 278.39 & $1.80 \times 10^{-2}$ & $2.77 \times 10^{-3}$ \\
4 & 448.59 & $2.21 \times 10^{-2}$ & $2.98 \times 10^{-5}$ & 448.59 & $2.21 \times 10^{-2}$ & $2.08 \times 10^{-3}$ \\
5 & 651.94 & $2.58 \times 10^{-2}$ & $2.17 \times 10^{-5}$ & 651.94 & $2.58 \times 10^{-2}$ & $8.52 \times 10^{-4}$ \\
6 & 884.80 & $2.9 \times 10^{-2}$ & $2.42 \times 10^{-5}$ & 884.80 & $2.9 \times 10^{-2}$ & $8.43 \times 10^{-4}$ \\
\hline
\end{tabular}

Table 4: Equivalent frequencies and associated loss factors of a clamped-clamped five layered sandwich structure 
Core in ISD112. A clamped-free beam with elastic faces in aluminium and core in 3M ISD112 is considered. A Generalized Maxwell model is used to model the frequency dependent shear modulus of the 3M ISD112 at the temperature of $27^{\circ}$ as follows:

$$
E(\omega)=E_{0}\left(1+\sum_{j=1}^{3} \frac{\Delta_{j} \omega}{\omega-i \Omega_{j}}\right)
$$

$E_{0}$ is the delayed elasticity of the shear modulus, $\left(\Delta_{j}, \Omega_{j}\right)$ are curve fitted-parameters. The different values of the fitted-parameters are resumed in table(5). The geometrical and ma-

\begin{tabular}{cccc}
\hline & & $27^{\circ}$ & \\
$\mathrm{j}$ & $E_{0}(\mathrm{~Pa})$ & $\Delta_{j}$ & $\Omega_{j}\left({\mathrm{rad} . \mathrm{s}^{-1}}^{6}\right.$ \\
\hline 1 & $0.5 \times 10^{6}$ & 0.746 & 468.7 \\
2 & & 3.265 & 4742.4 \\
3 & & 43.284 & 71532.5 \\
\hline
\end{tabular}

Table 5: Fit parameters for 3M ISD112 model

terial properties of the structure are presented in table 6: The free frequencies and the

\begin{tabular}{lcr}
\hline Elastic faces & Young modulus & $E_{f}=69 \times 10^{9} \mathrm{~Pa}$ \\
& Poisson ratio & $\nu=0.3$ \\
& Mass density & $\rho_{f}=2766 \mathrm{Kg} \cdot \mathrm{m}^{-3}$ \\
& Thickness & $h_{f}=1.524 \mathrm{~mm}$ \\
\hline Viscoelastic core & Poisson ratio & $\nu=0.5$ \\
& Mass density & $\rho_{c}=1600 \mathrm{Kg} \cdot \mathrm{m}^{-3}$ \\
& Thickness & $h_{c_{1}}=h_{c_{2}}=0.0423 \mathrm{~mm}$ \\
\hline Beam & Length & $\mathrm{L}=177.8 \mathrm{~mm}$ \\
& Width & $\mathrm{l}=12.7 \mathrm{~mm}$ \\
\hline
\end{tabular}

Table 6: Geometrical and material properties of a clamped-free five layered sandwich beam.

associated loss factor has been calculated and the results are presented in the table(7) The results of our model match perfectly those of [27].

\subsection{Structures with multilayered core}

\subsubsection{Constant viscoelastic model}

We consider a clamped-free structure whose viscoelastic core is composed of two different materials. The core is assumed to be made of two polymers with different loss factors $\{0.1 ; 0.5\}$ and same static Young modulus. The elastic faces are made in aluminium. The geometrical and material properties of the structure are presented in table8. The free frequencies and associated loss factors have been compared to those obtained by the method of 


\begin{tabular}{ccccccc}
\hline Five & layered & beam & & Three & layered & beam \\
\hline Modes & Frequency $(\mathrm{Hz}) \mathrm{s}$ & Loss factor & Error & Frequency $(\mathrm{Hz})$ & Loss factor & Error \\
\hline 1 & 65.23 & $1.71 \times 10^{-1}$ & $7.7 \times 10^{-4}$ & 65.23 & $1.71 \times 10^{-1}$ & $5.67 \times 10^{-4}$ \\
2 & 323.30 & $3.04 \times 10^{-1}$ & $1.36 \times 10^{-3}$ & 323.30 & $3.04 \times 10^{-1}$ & $1.36 \times 10^{-3}$ \\
3 & 846.82 & $3.32 \times 10^{-1}$ & $2.29 \times 10^{-3}$ & 846.82 & $3.32 \times 10^{-1}$ & $1.61 \times 10^{-3}$ \\
4 & 1555.3 & $3.15 \times 10^{-1}$ & $2.29 \times 10^{-3}$ & 1555.3 & $3.15 \times 10^{-1}$ & $1.66 \times 10^{-3}$ \\
5 & 2490.3 & $3.03 \times 10^{-1}$ & $2.34 \times 10^{-3}$ & 2490.3 & $3.03 \times 10^{-1}$ & $1.69 \times 10^{-3}$ \\
6 & 3671.2 & $2.88 \times 10^{-1}$ & $2.35 \times 10^{-3}$ & 3671.2 & $2.88 \times 10^{-1}$ & $1.70 \times 10^{-3}$ \\
\hline
\end{tabular}

Table 7: Equivalent frequencies and associated loss factors of a clamped-free five layered sandwich structure Aluminium/3MISD112 / Aluminium.

\begin{tabular}{ccc}
\hline Elastic faces & Young Modulus & $E_{f}=69 \times 10^{9} \mathrm{~Pa}$ \\
& Poisson ratio & $\nu=0.3$ \\
& Mass density & $\rho_{f}=2766 \mathrm{Kg}^{-3}$ \\
& Thickness & $h_{f}=1.524 \mathrm{~mm}$ \\
\hline Viscoelastic core & Young modulus & $E_{0}=1794 \times 10^{3} \mathrm{~Pa}$ \\
& Poisson ratio & $\nu=0.3$ \\
& Mass density & $\rho_{c}=968.1 \mathrm{Kg}^{-3}$ \\
& Thickness & $h_{c_{1}}=h_{c_{2}}=4.23 \times 10^{-4}$ \\
\hline Beam & Length & $\mathrm{L}=177.8 \mathrm{~mm}$ \\
& width & $\mathrm{l}=12.7 \mathrm{~mm}$ \\
\hline
\end{tabular}

Table 8: Geometrical and material properties of a five layered structure with heterogeneous core aluminium/polymères/aluminium. 
$\mathrm{Xu}$ et al. [28]. The results are presented in table 9. The model's results matches with those presented in [28], which validates our model for multi-layered viscoelastic cores.

\begin{tabular}{cccccc}
\hline \multicolumn{3}{c}{ Present model } & \multicolumn{3}{c}{ Layerwise } \\
\hline Modes & Frequency (Hz) & Loss factor & Error & Frequency $(\mathrm{Hz})$ & Loss factor \\
\hline 1 & 64.57 & $5.9 \times 10^{-} 2$ & $4.33 \times 10^{-4}$ & 64.77 & $5.9 \times 10^{-2}$ \\
2 & 298.09 & $5.24 \times 10^{-} 2$ & $1.36 \times 10^{-3}$ & 298.94 & $5.2 \times 10^{-2}$ \\
3 & 746.47 & $3.38 \times 10^{-} 2$ & $2.29 \times 10^{-3}$ & 748.26 & $3.35 \times 10^{-2}$ \\
4 & 1397.7 & $1.96 \times 10^{-} 2$ & $2.29 \times 10^{-3}$ & 1400.5 & $1.94 \times 10^{-2}$ \\
\hline
\end{tabular}

Table 9: Present model versus layerwise model of [28] for a clamped-free five layered sandwich structure with heterogeneous core (aluminium-polymers-aluminium) and validation

\section{Results}

The developed finite elements model is used to analyse a laminated glass structure similar to that described in [23]. The structure is made of two glass layers sandwiching a viscoelastic core made of acoustic PVB and PVB. The association of these two viscoelastic materials allows to have improved damping capabilities along with reasonable mechanical rigidity characteristics. The geometrical and material characteristics of the beam are recalled in Table 10. The Young's modulus of acoustic PVB is supposed to be complex constant. It is given

\begin{tabular}{lcr}
\hline Elastic Faces & Young Modulus & $E_{f}=64.5 \mathrm{GPa}$ \\
& Poisson ratio & $\nu=0.22$ \\
& Mass density & $\rho_{f}=2737 \mathrm{Kg} \cdot \mathrm{m}^{-3}$ \\
& Thickness & $h_{c_{1}}=1.4 \mathrm{~mm}$ \\
\hline PVB acoustic & Shear modulus & $G_{0}=0.7 \mathrm{MPa}$ \\
& Loss ratio & $\eta_{c_{2}}=0.9$ \\
& Poisson ratio & $\nu_{c_{2}}=0.49$ \\
& Mass density & $\rho_{c_{2}}=1000 \mathrm{Kg} \cdot \mathrm{m}^{-3}$ \\
& Thickness & $h_{c_{1}}=0.4 \mathrm{~mm}$ \\
\hline PVB & Shear modulus & $G_{0}=0.479 \mathrm{MPa}$ \\
& Poisson ratio & $\nu_{c_{3}}=0.4$ \\
& Mass density & $\rho_{c_{3}}=999 \mathrm{Kg} \cdot \mathrm{m}^{-3}$ \\
& Thickness & $h_{c_{2}}=1.4 \mathrm{~mm}$ \\
\hline Beam & Length & $L=1 \mathrm{~m}$ \\
& Width & $l=0.1 \mathrm{~m}$ \\
& Ref. Length & $h_{r e f}=5 \mathrm{~mm}$ \\
\hline
\end{tabular}

Table 10: Geometrical and material properties of the clamped-clamped beam

by the constitutive law :

$$
E_{2}(\omega)=2\left(1+\nu_{c_{2}}\right) G_{0}\left(1+i \eta_{c_{2}}\right)
$$


The Young's modulus of PVB is frequency and temperature dependent. At 20 degrees Celsius, it is given by the constitutive law :

$$
E_{3}(\omega)=2\left(1+\nu_{c_{3}}\right)\left(G_{\infty}+\left(G_{0}-G_{\infty}\right)\left(1+(i \omega \tau)^{1-\alpha}\right)^{-b}\right)
$$

with $G_{\infty}=2.3510^{8} \mathrm{~Pa}, \tau=0.3979 \mathrm{~s}, \alpha=0.46$ and $b=0.1946$ [29]. A clamped-clamped boundary condition is adopted and 200 elements are used to discretize the beam. Two test configurations are considered. The first one, denoted by $U C_{1}$, is characterized by a viscoelastic core made of a PVB layer interleaved between two acoustic PVB layers. The second one, denoted by $U C_{2}$ is characterized by a viscoelastic core made of an acoustic PVB layer sandwiched between two PVB layers. The adimensional thicknesses $X_{1}=\frac{h_{f}}{h_{r e f}}$ (elastic

faces), $X_{2}=\frac{h_{c_{1}}}{h_{r e f}}$ (acoustic PVB layer) and $X_{3}=\frac{h_{c_{2}}}{h_{r e f}}$ (PVB layer) are varied between 0.04 and 0.4. The quantities of interest are the damping of the first mode, the first resonant frequency and the rigidity index of the beam. We would like to see how these evolve versus $X_{1}, X_{2}$ and $X_{3}$.

\subsection{First case UC1}

The core is made up viscoelastic of an acoustic PVB layer (called Visco 2) sandwiched by two PVB layers (called Visco 1). In Figure 2, we fix $X_{1}=0.28$ (which corresponds to an elastic layer of $1.4 \mathrm{~mm}$ ) and we plot the previous quantities in function of $X_{2}$ (adimensional thickness of PVB layer) and $X_{3}$ (adimensional thickness of acoustic PVB). One can notice from Figure 2, that both viscoelastic layers thicknesses contribute to the damping. A closer look shows that the increase in damping is more influenced by the acoustic PVB layer than the PVB one which is quite normal given that the acoustic PVB has higher damping capacities than PVB. Moreover the rigidity index seems to be a linear function of each viscoelastic layer thickness which is quite logical since an increase in thickness increases the overall sandwich rigidity. The frequency exhibits a quadratic behaviour versus the acoustic PVB layer and a linear behaviour versus the PVB layer. In Figure 3, we fix $X_{3}=0.28$ (which corresponds to a central viscoelastic layer of $1.4 \mathrm{~mm}$ ) and we plot the previous quantities in function of $X_{1}$ (adimensional thickness of elastic layer) and $X_{2}$ (adimensional thickness of PVB layer). It is obvious that the thickness of the faces has a stronger impact than the thickness of the PVB layer on the damping. Moreover, one can see easily that there is an optimal face thickness for which the damping is maximized. Moreover, it is worth noting that for the frequency and rigidity if the viscoelastic thickness contribute in a linear fashion, the elastic face thickness seems to contribute quadratically. In Figure 4, we fix $X_{2}=0.08$ (which corresponds to an elastic layer of $0.4 \mathrm{~mm}$ ) and we plot the previous quantities in function of $X_{1}$ and $X_{3}$, the adimensional viscoelastic layers thicknesses. In this last figure, one can see that the faces thickness and acoustic PVB layer thickness contribute equally to the damping. The existence 

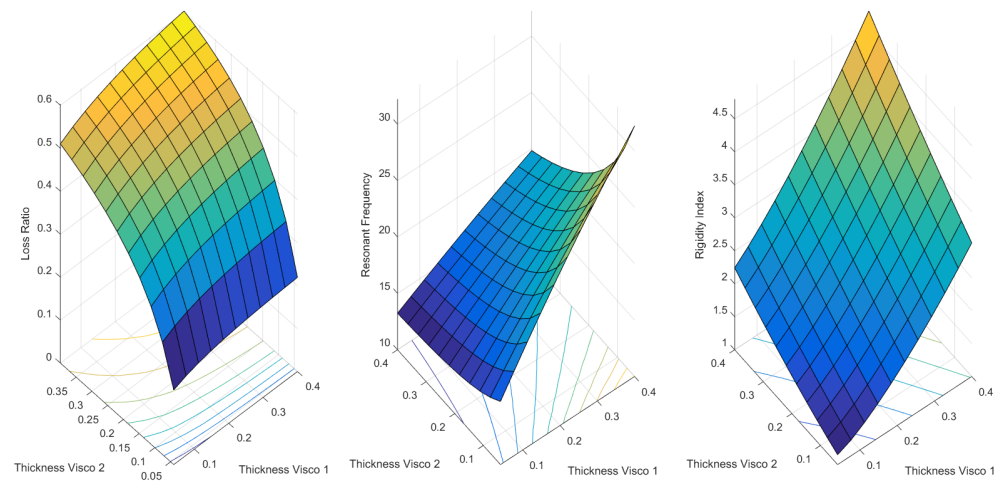

Figure 2: Evolution of loss ratio, resonant frequency and rigidity index versus $X_{2}, X_{3}$ for $X_{1}=0.28$
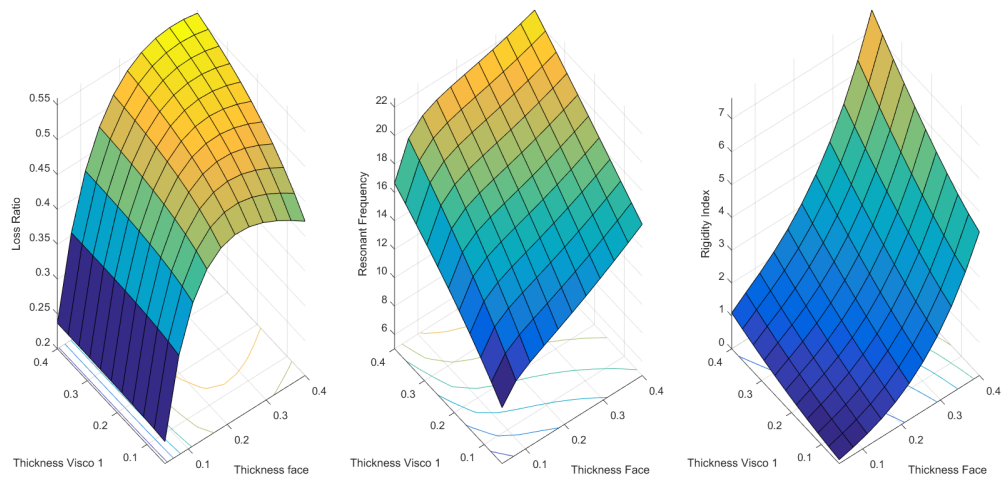

Figure 3: Evolution of loss ratio, resonant frequency and rigidity index versus $X_{1}, X_{2}$ for $X_{3}=0.28$ 
of an optimal face thickness that maximizes the damping is also observed as in Figure 3. The frequency shows the same quadratic behaviour versus the acoustic PVB layer as noted on Figure 2 and linear behaviour versus the face thickness as observed on Figure 3. A quick comparison of Figures 2, 3 and 4 shows that face thickness, PVB layer thickness and acoustic PVB layer thickness contribute in decreasing order to the rigidity index which quite logical given their respective Young's moduli.
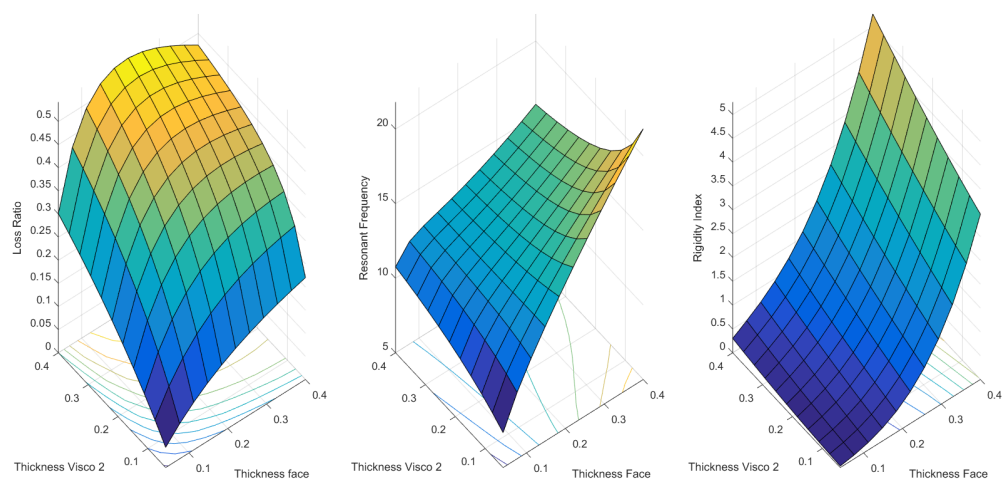

Figure 4: Evolution of loss ratio, resonant frequency and rigidity index versus $X_{1}, X_{3}$ for $X_{2}=0.08$

\subsection{Second case UC2}

The core is made up viscoelastic of a PVB layer sandwiched by two acoustic PVB layers. In Figure 5, we fix $X_{1}=0.28$ (which corresponds to an elastic layer of $1.4 \mathrm{~mm}$ ) and we plot the previous quantities in function of $X_{2}$ (acoustic PVB layer) and $X_{3}$ (PVB layer), the adimensional viscoelastic layers thicknesses. One can notice that both viscoelastic layers thicknesses contribute to the damping, the acoustic PVB layer thickness having more influence than the PVB layer one as observed previously on Figure 2. As observed on Figure 2, the frequency seems to be quadratic versus the acoustic PVB layer thickness while being linear versus the PVB layer thickness. The rigidity index evolves in a linear fashion versus the two viscoelastic layers' thicknesses. In Figure 6, we fix $X_{3}=0.28$ (which corresponds to a central viscoelastic layer of $1.4 \mathrm{~mm}$ ) and we plot the previous quantities in function of $X_{1}$ and $X_{2}$, the adimensional viscoelastic layers thicknesses. One can notice that there is an optimal thickness of the faces that maximizes the damping while it increases in function of the thickness of the acoustic PVB layer. The linearity of the frequency versus the thickness of the elastic faces and its quadratic behaviour versus the acoustic PVB layer thickness is recovered as observed before in Figure 3. The rigidity index seems to be quadratic in function of the elastic faces thickness and linear versus the acoustic PVB layer thickness. In Figure 7, we fix $X_{2}=0.08$ (which corresponds to a central viscoelastic layer of $0.4 \mathrm{~mm}$ ) and we 

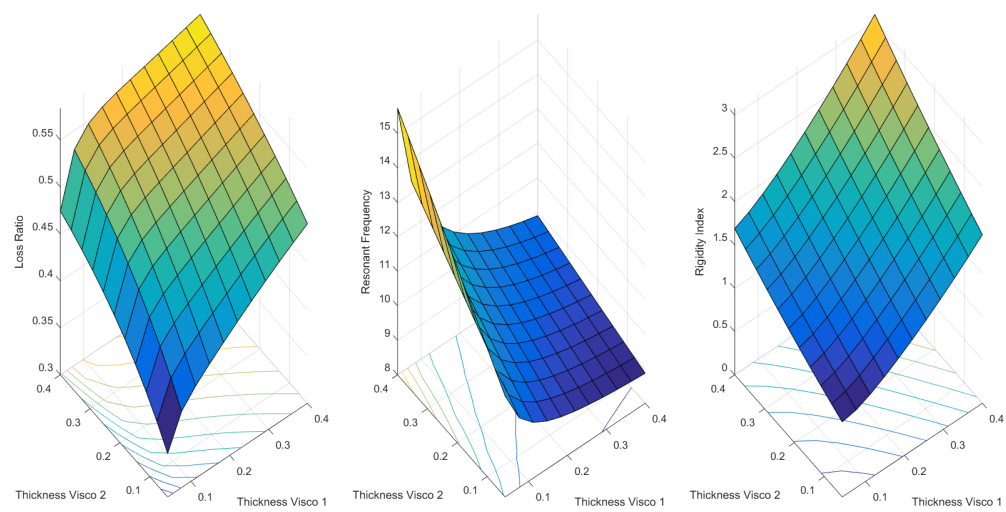

Figure 5: Evolution of loss ratio, resonant frequency and rigidity index versus $X_{2}, X_{3}$ for $X_{1}=0.28$
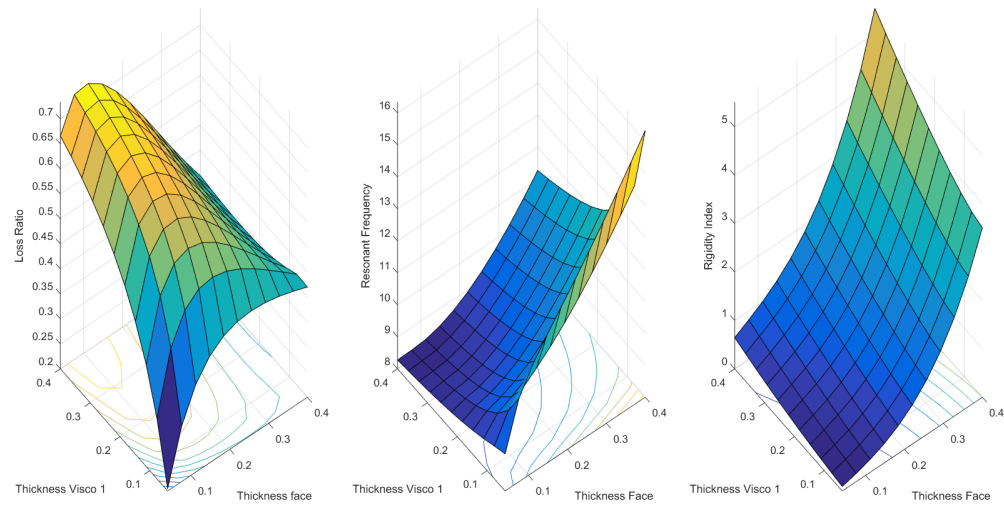

Figure 6: Evolution of loss ratio, resonant frequency and rigidity index versus $X_{1}, X_{2}$ for $X_{3}=0.28$ 
plot the previous quantities in function of $X_{1}$ and $X_{3}$, the adimensional viscoelastic layers thicknesses. One can notice that the PVB layer thickness does not affect much the damping. The frequency and rigidity index seem to be linear in function this quantity.
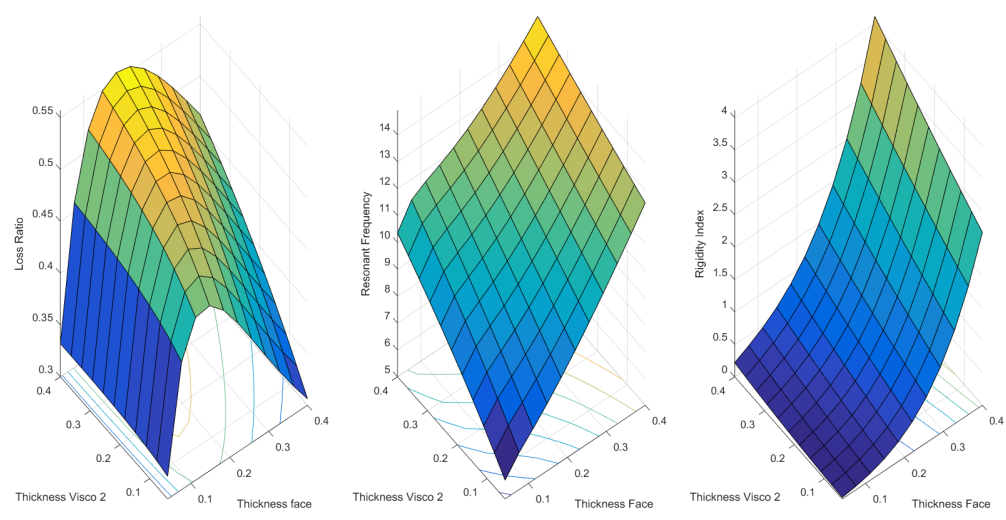

Figure 7: Evolution of loss ratio, resonant frequency and rigidity index versus $X_{1}, X_{3}$ for $X_{2}=0.08$

\section{Conclusion}

In the present article, a computational model of a five layered sandwich beam with a multi-layered viscoelastic core is presented and validated for frequency dependent viscoelastic materials. The model is then used to perform a parametric study of a laminated glass beam with PVB acoustic and PVB as damping viscoelastic materials. Some relevant quantities such as the damping, the resonant frequency and the rigidity of the beam are investigated in function of the thicknesses. It is shown in particular that if the rigidity is quadratic in function of the elastic thickness and linear in function of the viscoelastic thicknesses, the damping and resonant frequency show non monotonous variations. In particular, the existence of an optimal faces thickness for damping is shown while a quadratic behaviour of frequency versus acoustic PVB layer thickness is reported. In perspectives, it can be interesting to extend the present study to higher order modes, especially in the presence of interacting modes. Moreover, multi-criteria optimization can be used to determine the compromises between the design parameters to achieve the highest performances in damping and rigidity for low mass sandwich structures.

\section{Acknowledgment}

The authors would like to acknowledge LABEX DAMAS (Lorraine) for their financial support and all the anonymous reviewers. 
Appendix .1. Shape functions and element matrices

Denoting by $L^{e}$ the element length and setting $\xi=\frac{2 x}{L^{e}}-1$ for $x \in\left[0, L^{e}\right]$ and $\xi=[-1,1]$, the classical polynomial shape functions are written as follows:

$$
\begin{aligned}
& n_{1}(\xi)=\frac{(1-\xi)^{2}(2+\xi)}{4}, \\
& n_{2}(\xi)=\frac{L^{e}(1-\xi)^{2}(1+\xi)}{8}, \\
& n_{3}(\xi)=\frac{(1+\xi)^{2}(2-\xi)}{4}, \\
& n_{4}(\xi)=\frac{L^{e}(1+\xi)^{2}(1-\xi)}{8}, \\
& n_{5}(\xi)=\frac{1-\xi}{2}, \\
& n_{6}(\xi)=\frac{1-\xi}{2}, \\
& n_{7}(\xi)=\frac{1+\xi}{2}, \\
& n_{8}(\xi)=\frac{1+\xi}{2} .
\end{aligned}
$$

The resulting shape functions matrices listed in section 3 can then be written as:

$$
\begin{aligned}
& {\left[N_{w}\right]=\left[\begin{array}{llllllll}
n_{1}(\xi) & n_{2}(\xi) & 0 & 0 & n_{3}(\xi) & n_{4}(\xi) & 0 & 0
\end{array}\right] ;} \\
& {\left[N_{\beta_{2}}\right]=\left[\begin{array}{llllllll}
0 & 0 & n_{5}(\xi) & 0 & 0 & 0 & n_{6}(\xi) & 0
\end{array}\right] ;} \\
& {\left[N_{\beta_{3}}\right]=\left[\begin{array}{llllllll}
0 & 0 & 0 & n_{7}(\xi) & 0 & 0 & 0 & n_{8}(\xi)
\end{array}\right] .}
\end{aligned}
$$

As for the element mass $[\mathrm{Me}]$ and the element stiffness $[\mathrm{Ke}]$ mentioned in section 3, they can be expressed as:

$$
\left[M_{e}\right]=\left(2 \rho_{f} S_{f}+3 \rho_{c_{i}} S_{c_{i}}\right) \int_{-1}^{1} J^{t}\left[N_{w}\right]\left[N_{w}\right] d \xi .
$$




$$
\begin{aligned}
K_{e v_{2}}= & \frac{S_{c_{2}}}{\left(1+\nu_{c_{2}}\right)}\left[k_{7}^{e}\right]+\left(2 I_{c_{2}}+\frac{1}{2} h_{c_{1}}^{2} S_{c_{2}}\right)\left[k_{1}^{e}\right] \\
& +\frac{1}{2} S_{c_{2}} h_{c_{1}} h_{c_{2}}\left[k_{2}^{e}\right]+\frac{1}{2} h_{c_{2}}^{2} S_{c_{2}}\left[k_{4}^{e}\right] \\
K_{e v_{3}}= & I_{c_{3}}\left[k_{4}^{e}\right]+\frac{S_{c_{3}}}{2\left(1+\nu_{c_{3}}\right)}\left[k_{8}^{e}\right] \\
K_{e_{0}}= & E_{f} S_{f} h_{c_{1}}\left(2 h_{c_{1}}\left[k_{1}^{e}\right]+h_{c_{2}}\left[k_{2}^{e}\right]-h_{f}\left[k_{3}^{e}\right]\right) \\
& +\frac{1}{2} E_{f} S_{f} h_{c_{2}}\left(h_{c_{2}}\left[k_{4}^{e}\right]-h_{f}\left[k_{5}^{e}\right]\right) \\
& +E_{f}\left(2 I_{f}+\frac{1}{2} S_{f} h_{f}^{2}\right)\left[k_{6}^{e}\right] \\
& +\frac{E_{2}(0)}{2\left(1+\nu_{c_{2}}\right)} K_{e v_{2}}+\frac{E_{3}(0)}{2\left(1+\nu_{c_{3}}\right)} K_{e v_{3}} \\
K_{e}(\omega)= & K_{e_{0}}+\left(E_{2}(\omega)-E_{2}(0)\right) K_{e v_{2}}+\left(E_{3}(\omega)-E_{3}(0)\right) K_{e v_{3}}
\end{aligned}
$$

with $E_{i}(\omega)$ the frequency dependent Young's modulus of the viscoelastic material of layer $i$.

$$
\begin{gathered}
\left\{\begin{array}{l}
{\left[k_{1}^{e}\right]=\int_{-1}^{1} J^{-1}\left[N_{\beta_{2}}^{\prime}\right]^{t}\left[N_{\beta_{2}}^{\prime}\right] d \xi} \\
{\left[k_{2}^{e}\right]=\int_{-1}^{1} J^{-1}\left(\left[N_{\beta_{2}}^{\prime}\right]^{t}\left[N_{\beta_{3}}^{\prime}\right]+\left[N_{\beta_{3}}^{\prime}\right]^{t}\left[N_{\beta_{2}}^{\prime}\right]\right) d \xi} \\
{\left[k_{3}^{e}\right]=\int_{-1}^{1} J^{-2}\left(\left[N_{\beta_{2}}^{\prime}\right]^{t}\left[N_{w}^{\prime \prime}\right]+{ }^{t}\left[N_{w}^{\prime \prime}\right]\left[N_{\beta_{2}}^{\prime}\right]\right) d \xi} \\
{\left[k_{4}^{e}\right]=\int_{-1}^{1} J^{-1}\left[N_{\beta_{3}}^{\prime}\right]^{t}\left[N_{\beta_{3}}^{\prime}\right] d \xi}
\end{array}\right. \\
\left\{\begin{array}{l}
{\left[k_{5}^{e}\right]=\int_{-1}^{1} J^{-2}\left(\left[N_{\beta_{3}}^{\prime}\right]^{t}\left[N_{w}^{\prime \prime}\right]+\left[N_{w}^{\prime \prime}\right]^{t}\left[N_{\beta_{3}}^{\prime}\right]\right) d \xi} \\
{\left[k_{6}^{e}\right]=\int_{-1}^{1} J^{-3}\left(\left[N_{w}^{\prime \prime}\right]^{t}\left[N_{w}^{\prime \prime}\right]\right) d \xi} \\
{\left[k_{7}^{e}\right]=\int_{-1}^{1}\left(\left[N_{\beta_{2}}\right]^{t}\left[N_{w}^{\prime}\right]+J\left[N_{\beta_{2}}\right]^{t}\left[N_{\beta_{2}}\right]+J^{-1}\left[N_{w}^{\prime}\right]^{t}\left[N_{w}^{\prime}\right]+\left[N_{w}^{\prime}\right]^{t}\left[N_{\beta_{2}}\right]\right) d \xi} \\
{\left[k_{8}^{e}\right]=\int_{-1}^{1}\left(\left[N_{\beta_{3}}\right]^{t}\left[N_{w}^{\prime}\right]+J\left[N_{\beta_{3}}\right]^{t}\left[N_{\beta_{3}}\right]+J^{-1}\left[N_{w}^{\prime}\right]^{t}\left[N_{w}^{\prime}\right]+\left[N_{w}^{\prime}\right]^{t}\left[N_{\beta_{3}}\right]\right) d \xi}
\end{array}\right.
\end{gathered}
$$

where $J=L^{e} / 2$.

Appendix .2. Axial force and flexural moment

The expression of the axial forces and the flexural moment is deduced from the constitutive behaviour of the five layered sandwich beam (based on the generalised Hooke's stress-strain law).

$$
\left\{\begin{array}{l}
N_{i}(x, t)=\iint_{s_{i}} \sigma_{i} d s=E_{f} S_{f}\left(u_{i}^{\prime}+\frac{1}{2} w^{\prime 2}\right) \quad i=1,5 \\
N_{i}(x, t)=\iint_{s_{i}} \sigma_{i} d s=S_{c_{i}} Y_{i}(t) * \frac{\partial}{\partial t}\left(u_{i}^{\prime}+\frac{1}{2} w^{\prime 2}\right) \quad i=2,3,4
\end{array}\right.
$$


where $S_{f}$ and $S_{c_{i}}$ denote the surface area of the elastic layer and the viscoelastic layer $i$, respectively. The bending moment is written as follows:

$$
\left\{\begin{array}{l}
M_{i}=E_{f} S_{f} \ddot{w} \quad i=1,5, \\
M_{i}=I_{c_{i}} Y_{i} * \beta_{i}{ }^{\prime} \quad i=2,3,4
\end{array}\right.
$$

where $E_{f}, I_{c_{i}}$ and $Y_{i}$ are respectively the Young's modulus, the quadratic moment of the layers' cross section and the relaxation function. The shear force $T_{i}$ in the viscoelastic layers $i=(2,3,4)$ can be written as :

$$
T_{i}=\iint_{s_{i}} \tau_{i} d s=\frac{S_{c_{i}}}{2\left(1+\nu_{c_{i}}\right)} Y_{i}(t) * \frac{\partial}{\partial t}\left(\frac{\partial w}{\partial x}+\beta_{i}\right) .
$$

with $\tau_{i}=\frac{Y_{i}(t)}{2\left(1+\nu_{c_{i}}\right)} * \frac{\partial \xi_{i}}{\partial t}$ is the shear stress, $\nu_{c_{i}}$ the Poisson ratio of viscoelastic layer $i$.

\section{References}

[1] K. E.M., Damping of flexural waves by a constrained viscoelastic layer, Journal of the Acoustic Society of America 31 (7) (1959) 952-962.

[2] E.E.Ungar, Loss factor of viscoelastically damped beam structures, The Journal of the Acoustical Society of America 34 (1962) 1082-1089.

[3] R. A. DiTaranto, W. Blasingame, Composite damping of vibrating sandwich beams, Journal of Engineering for Industry 89 (1967) 633-638. doi:http://dx.doi.org/10.1115/1.3610124.

[4] Mead, Markus, The forced vibration of a three-layer, damped sandwich beam with arbitrary boundary conditions, Journal of Sound and Vibration 10 (2) (1969) 163 - 175. doi:http://dx.doi.org/10.1016/0022-460X(69)90193-X.

[5] D. K. Rao, Frequency and loss factors of sandwich beams under various boundary conditions, Journal of Mechanical Engineering Science 20 (5) (1978) 271-282. arXiv:http://jms.sagepub.com/content/20/5/271.full.pdf + html, doi:10.1243/JMES_JOUR_1978_020_047_02.

[6] M.L.Soni, Finite element analysis of viscoelastically damped sandwich structures, Shock and Vibration Bulletin 55 (1) (1981) 97-109. 
[7] B.-A. Ma, J.-F. He, A finite element analysis of viscoelastically damped sandwich plates, Journal of Sound and Vibration 152 (1) (1992) 107 - 123. doi:http://dx.doi.org/10.1016/0022-460X(92)90068-9.

[8] R. Rikards, A. Chate, E. Barkanov, Finite element analysis of damping the vibrations of laminated composites, Computers \& Structures 47 (6) (1993) 1005 - 1015. doi:http://dx.doi.org/10.1016/0045-7949(93)90305-W.

[9] E. M. Daya, L. Azrar, M. Potier-Ferry, Modélisation par éléments finis des vibrations non-linéaires des plaques sandwich viscoélastiques, Mechanics \& Industry 6 (2005) 1320. doi:10.1051/meca:2005002.

[10] J. Moita, A. Araujo, P. Martins, C. M. Soares, C. M. Soares, A finite element model for the analysis of viscoelastic sandwich structures, Computers \& Structures 89 (21-22) (2011) 1874 - 1881. doi:http://dx.doi.org/10.1016/j.compstruc.2011.05.008.

[11] G. Jin, C. Yang, Z. Liu, Vibration and damping analysis of sandwich viscoelastic-core beam using reddy's higher-order theory, Composite Structures 140 (2016) 390 - 409. doi:http://dx.doi.org/10.1016/j.compstruct.2016.01.017.

[12] M. F. C. Jr., A. J. Ferreira, V. Tita, A review on plate and shell theories for laminated and sandwich structures highlighting the finite element method, Composite Structures (2016) -doi:http://dx.doi.org/10.1016/j.compstruct.2016.02.036.

[13] C. D. Johnson, D. A. Kienholz, Finite element prediction of damping in structures with constrained viscoelastic layers, American Institute of Aeronautics and Astronautics 20 (9) (1982) 1284-1290.

[14] E. M. Daya, M. Potier-Ferry, A numerical method for nonlinear eigenvalue problems application to vibrations of viscoelastic structures, Computers \& Structures 79 (5) (2001) 533-541.

[15] Y.Koutsawa, I.Charpentier, E.M.Daya, M.Cherkaoui, A generic approach for the solution of nonlinear residual equations. part i: The diamant toolbox., Computer Methods in Applied Mechanics and Engineering 198 (3-4) (2008) 572-577.

[16] A. Araújo, C. Mota Soares, C. Mota Soares, Finite element model for hybrid activepassive damping analysis of anisotropic laminated sandwich structures, Journal of Sandwich Structures and Materials 12 (4) (2010) 397-419. doi:10.1177/1099636209104534.

[17] J. Li, Y. Narita, Analysis and optimal design for the damping property of laminated viscoelastic plates under general edge conditions, Composites Part B: Engineering 45 (1) (2013) 972 - 980. doi:http://dx.doi.org/10.1016/j.compositesb.2012.09.014. 
[18] S.-N. Nguyen, J. Lee, M. Cho, Efficient higher-order zig-zag theory for viscoelastic laminated composite plates, International Journal of Solids and Structures 62 (2015) $174-$ 185. doi:http://dx.doi.org/10.1016/j.ijsolstr.2015.02.027.

[19] C. Yang, G. Jin, Z. Liu, X. Wang, X. Miao, Vibration and damping analysis of thick sandwich cylindrical shells with a viscoelastic core under arbitrary boundary conditions, International Journal of Mechanical Sciences 92 (2015) 162-177. doi:http://dx.doi.org/10.1016/j.ijmecsci.2014.12.003.

[20] K. Akoussan, H. Boudaoud, E.-M. Daya, E. Carrera, Vibration modeling of multilayer composite structures with viscoelastic layers, Mechanics of Advanced Materials and Structures 22 (1-2) (2015) 136-149. doi:10.1080/15376494.2014.907951.

[21] K. Bouayed, M.-A. Hamdi, Finite element analysis of the dynamic behavior of a laminated windscreen with frequency dependent viscoelastic core, Journal of the acoustical societey of America 132 (2) (2012) 757-766. doi:10.1121/1.4733554.

[22] M. Rehfeld, D. Fournier, J. Boure, C. Payen, Viscoelastic plastic interlayer for vibroacoustic damping and glazing comprising such an interlayer, uS Patent App. 13/495,018 (Nov. 28 2013).

URL \url\{https://www.google.com/patents/US20130316158\}

[23] M. Friedman, D. McDonnell-Lee, M. Rehfeld, J. Bravet, J. Boure, V. Offermann, Transparent acoustical and mechanical barrier, uS Patent 6,432,522 (Aug. 13 2002).

URL \url\{https://www.google.fr/patents/US6432522\}

[24] M. Bilasse, L. Azrar, E. Daya, Complex modes based numerical analysis of viscoelastic sandwich plates vibrations, Computers \& Structures 89 (7-8) (2011) 539 - 555. doi:http://dx.doi.org/10.1016/j.compstruc.2011.01.020.

[25] X. Chen, H. Chen, X. Hu, Damping prediction of sandwich structures by orderreduction-iteration approach, Journal of Sound and Vibration 222 (5) (1999) 803 - 812. doi:http://dx.doi.org/10.1006/jsvi.1998.2131.

[26] T. G. Dhatt G., Lefrancois E., Finite element method, Numerical methods series, Wiley, 2012.

[27] M. Bilasse, I. Charpentier, E. M. Daya, Y. Koutsawa, A generic approach for the solution of nonlinear residual equations. part ii: Homotopy and complex nonlinear eigenvalue method, Computer Methods in Applied Mechanics and Engineering 198 (49-52) (2009) 3999 - 4004. doi:http://dx.doi.org/10.1016/j.cma.2009.09.015. 
[28] C. Xu, S. Lin, Y. Yang, Optimal design of viscoelastic damping structures using layerwise finite element analysis and multi objective generic algorithm, Computers \& Structures 157 (2015) 1-8.

[29] Haberman, Design of high loss viscoelastic composites through micromechanical modeling and decision based material by design, Ph.D. thesis, George W. Woodruff School of Mechanical Engineering, Atlanta (2007). 